\title{
Value-Based Analysis for Breast Cancer Treatment: We Don't Know What We Don't Know
}

\author{
Theresa L. Schwartz, MD, FACS ${ }^{1}$ and Julie A. Margenthaler, MD, FACS ${ }^{2}$ \\ ${ }^{1}$ Department of Surgery, St. Louis University, St. Louis, MO; ${ }^{2}$ Department of Surgery, Washington University School of \\ Medicine, St. Louis, MO
}

As we enter 2019, health care costs are at their highest, with only continued increases in store. In 2020, health care spending for cancer in the US is projected to reach at least $\$ 158$ billion $^{1}$, up $27 \%$ since 2010 . With breast cancer being the most common malignancy in women, it is paramount to investigate the costs associated with the local and systemic therapy options available for its treatment. New payment and delivery models are in the trial stages to improve the effectiveness and efficiency of specialty care, including the Oncology Care Model from the Centers for Medicare and Medicaid Services. With a goal of utilizing appropriately aligned financial incentives to enable improved care coordination, appropriateness of care, and access to care to beneficiaries undergoing chemotherapy at the same or lower cost as Medicare, the Oncology Care Model should also have significant implications for reimbursement. We are all well aware of the national use of quality measures as a surrogate for the delivery of high-quality care, but the majority of these will not necessarily affect us as they are not considered 'surgical' in nature. However, patient-reported outcomes can be directly affected by surgical oncology care, and the development of value-based metrics for surgical procedures is in its infancy.

As surgical oncologists, we need to take a more active role in not only understanding what these measures mean but also in determining their importance. We cannot afford to be bystanders and simply watch as quality of surgical care is defined. In their manuscript entitled A Systematic Review of Utility Score Assessments in the Breast Surgery

(C) Society of Surgical Oncology 2019

First Received: 5 January 2019;

Published Online: 23 January 2019

J. A. Margenthaler, MD, FACS

e-mail: margenthalerj@wudosis.wustl.edu
Cost-Analysis Literature, published in this issue of Annals of Surgical Oncology, Yoon et al. ${ }^{2}$ have added to the conversation by reporting on utility scores for both oncologic breast resections and reconstruction. However, in order to begin to comprehend their data and its potential applications, we must first have a handle on the concepts of the analyses. Cost-utility analyses, when performed correctly, can be very powerful tools as they describe both quality- and quantity-of-life expectations for a given treatment. Their complexity is just as high as the expected impact of their results.

First, what is a utility score? In health economics, utility measurement consists of two main components: (1) the definition of a set of health states of interest; and (2) the value of (i.e. preference for) each defined health state. The ways in which a health state can be defined, and the value derived, can vary dramatically from one paper to the next. As an example, one could define the health states for mastectomy as mastectomy with no complications versus mastectomy with postoperative hematoma. The value of each defined health state would then be determined according to the results of a questionnaire ideally given to affected patients, but which can also be given to health care providers, such as the visual analogue scale, in which a numerical value within a specific scale would be assigned. While these questionnaires can be significantly different from one another and are rarely described in detail in the article in which they are presented, they are generally filled with scenarios, and each scenario is ranked from 0 (representing death) to 1 (representing perfect health). One example could be: You have undergone a mastectomy and developed a hematoma that required operative drainage with a 2-night stay in the hospital followed by subsequent incisional breakdown necessitating wet to dry dressings at 
home for 3 weeks. The ranked scores for each scenario would then be pooled and averaged to get the final utility score. This utility score is what determines quality of life.

So, you have a set of utility scores to estimate quality of life. Now what? The next step is to calculate quality-adjusted life-years (QALYs) in order to combine quality of life with quantity of life. A QALY of 1 assumes a year of life in perfect health. In order to calculate QALYs, you first multiple the utility score of a given health state by the length of time spent in that state. This result is then added to the product of the remaining life expectancy for the patient minus the time spent in that state and the utility score of a health state without the described complication. See example calculation below estimating QALYs for a mastectomy complicated by a postoperative hematoma. This adjustment will level the playing field so that QALYs can be used to compare outcomes, treatments, complications, adverse effects, or any number of health states. perfect health, which is defined in the US as $\$ 50,000$. In other countries, such as the UK, the 'willingness to pay' is lower at $\$ 30,000 / \mathrm{QALY}$.

One of the most striking outcomes of the analysis by Yoon et al. ${ }^{2}$ is the relative paucity of robust data on the topic of utility scores for cancer-related breast surgical procedures. Only 19 studies met all the criteria for inclusion in this analysis. These researchers identified that utility scores within the breast-conserving therapy category yielded the highest mean utility score at 0.79 , compared with 0.76 in the mastectomy without reconstruction group. Mean utility scores were lower in reconstruction states, with autologous reconstruction outperforming implantbased reconstruction ( 0.73 vs. 0.64$)$. While these utility scores are important in establishing a foundation for cancer care cost analyses, there are other variables, such as actual time, within a health state, and the likelihood of being in that health state, that significantly impact the analyses.

Utility score of mastectomy with hematoma $\times$ duration of health state

$$
\mathrm{QALY}=\text { Utility score of uncomplicated mastectomy } \times \text { (remaining life years-duration of health state })
$$

For completion purposes, we should also briefly review how to begin assessment of cost utility, even though it was not examined in this article. You now have objective data to describe quality and quantity of life. How can this be translated into cost utility? It is time to calculate the incremental cost-utility ratio (ICUR) by subtracting the expected cost of one health state from the expected cost of another health state. This result is then divided by the total of the expected QALY from one health state subtracted from the QALY of another health state. ${ }^{3}$ See example calculation below estimating the ICUR for a mastectomy with implant reconstruction.
These variables will need to be quantitated in order to have meaningful data for comparison.

Given the potential ramifications of cost-utility analyses of breast cancer procedures, there should be a tremendous push towards expanding the available data and pooling results for future research. Creating a standardized list of health states for breast cancer-related operations, as well as questionnaires to determine the value of each health state, also appears to be necessary. To better compare one study's reported utility scores with another, having a more uniform method of calculating these scores seems essential. Additionally, as our breast cancer surgical procedures become

ICUR $=\frac{(\text { Expected cost of mastectomy without reconstruction }- \text { Expected cost of mastectomy with implant reconstruction })}{(\text { Expected QALY of mastectomy without reconstruction }- \text { Expected QALY of mastectomy with implant reconstruction })}$

The ICUR represents the added cost to prolong a patient's life by 1 year of perfect health. A treatment is considered cost effective if the ICUR is $>0$ and less than the standard 'willingness to pay' for an added year of more complex than the CPT codes may suggest (such as nipple-sparing mastectomy or oncoplastic breast conservation with the contralateral symmetry procedure), so too should our assessment of patient-centered quality of life and QALYs. Current reimbursement strategies infrequently 
consider the extra training to learn how to develop the surgical expertise or the added operative time to perform the procedures. In order to effectively influence health care policy in such a way as to impact reimbursement for more complex surgical techniques that require more time and skill, we, as surgeons, should be at the forefront of both assessing and optimizing patient-reported outcomes and identifying new ways to make value-based analysis of breast cancer treatment a thriving body of research.

\section{REFERENCES}

1. Centers for Medicare and Medicaid Services. https://innovation.c ms.gov/initiatives/Oncology-Care/. Accessed 1 Jan 2019.
2. Yoon AY, Bozzuto L, Seto AJ, Fisher CS, Chatterjee A. A systematic review of utility score assessments in the breast surgery cost-analysis literature. Ann Surg Oncol. 2019. https://doi.org/10. 1245/s10434-019-07160-x.

3. Krishnan NM Chatterjee A, Rosenkranz KM, Powell SG, Nigriny JF, Vidal DC. The cost effectiveness of acellular dermal matrix in expander-implant immediate breast reconstruction. J Plast Reconstr Aesthet Surg. 2014;67(4):468-476.

Publisher's Note Springer Nature remains neutral with regard to jurisdictional claims in published maps and institutional affiliations. 